\title{
Tailoring Crystal Structures and Polymorphs of Halogen-Bonded Supramolecular Assemblies: Co-Crystals of Hexahalogenated Benzenes and 2,3,5,6-Tetramethylpyrazine
}

\author{
R. K. El-khawaldeh, S. S. Gunaga, D. L. Bryce* \\ Department of Chemistry \& Biomolecular Sciences, University of Ottawa, Ottawa, Canada \\ relkh100@uottawa.ca
}

Polymorphism is of significant interest owing to its potential to influence the physiochemical properties of pharmaceuticals and other materials. The high directionality and the intrinsic tunability of the intermolecular halogen bond make it a compelling design element in crystal engineering. In this work, we describe various polymorphs of halogen-bonded co-crystals constructed from 2,3,5,6tetramethylpyrazine with different halogen bond donors (1,4-diiodotetrafluorobenzene, 1,3,5-trifluoro-2,4,6-triiodobenzene, and their bromoand chloro-analogues). Polymorphic cocrystals are obtained from solution-based and solid-based methods. Solution-based methods are implemented by manipulating the solvent system mixture and the mixing temperature while solid-based cocrystals are obtained via cosublimation and mechanochemical techniques. Co-crystal structures are characterized via single crystal X-ray diffraction and powder X-ray diffraction. 13C solid-state nuclear magnetic resonance spectroscopic investigations of cocrystals are used to validate and further probe the polymorphic structures. This study serves as a holistic approach in investigating polymorphism and demonstrates the unquenchable importance of halogen bonding in crystal engineering.

Keywords: Crystal Engineering; Halogen Bonding; Polymorphism. 